INPLASY

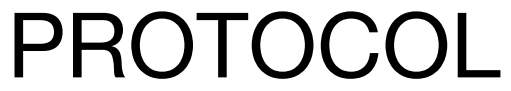

To cite: Chen et al. Multiple Antiarrhythmic Transplacental Treatments for Fetal

Supraventricular

Tachyarrhythmia: protocol for a systematic review and metaanalysis. Inplasy protocol

2020100063. doi:

10.37766/inplasy2020.10.0063

Received: 17 October 2020

Published: 17 October 2020

Corresponding author:

Xiao Yang

yczar@126.com

Author Affiliation:

Chengdu Women's and

Children's Central Hospital,

School of Medicine, University

of Electronic Science and

Technology of China

Support: National key

research.

Review Stage at time of this submission: The review has not yet started.

Conflicts of interest:

The authors have no conflicts of interest to disclose.

\section{Multiple Antiarrhythmic \\ Transplacental Treatments for Fetal Supraventricular Tachyarrhythmia: protocol for a systematic review and meta-analysis}

Chen, TT1; Yang, YF2; Shi, K³; Pan, Y4; Wei, SM5; Yang, ZX6; Yang $X^{7}$.

Review question / Objective: The primary aim of this study is to undertake a comprehensive systematic review and metaanalysis to evaluate the polytherapy for fetal supraventricular tachyarrhythmia, and tease out what factors influence their effectiveness.

Condition being studied: Fetal tachyarrhythmia can lead to fetal nonimmune hydrops, cardiac dysfunction, premature delivery, or even fetal demise. However, there is no standardized treatment of fetal supraventricular tachyarrhythmia.

Information sources: PubMed, EMBASE, Cochrane Library, Web of Science, Google Scholar and ClinicalTrials.gov will be searched from inception to September 2020. A handsearching for grey literature, including unpublished conference articles will be performed.

INPLASY registration number: This protocol was registered with the International Platform of Registered Systematic Review and Meta-Analysis Protocols (INPLASY) on 17 October 2020 and was last updated on 17 October 2020 (registration number INPLASY2020100063).

\section{INTRODUCTION}

Review question / Objective: The primary aim of this study is to undertake a comprehensive systematic review and meta-analysis to evaluate the polytherapy for fetal supraventricular tachyarrhythmia, and tease out what factors influence their effectiveness. 
Condition being studied: Fetal tachyarrhythmia can lead to fetal nonimmune hydrops, cardiac dysfunction, premature delivery, or even fetal demise. However, there is no standardized treatment of fetal supraventricular tachyarrhythmia.

\section{METHODS}

Participant or population: Patients with sustained SVT, or AFL, or patients with non-sustained fetal SVT with evidence of cardiac dysfunction, who are treated with multiple antiarrhythmic transplacental therapy.

Intervention: We define multiple antiarrhythmic transplacental treatments (digoxin, amiodarone, flecainide, or sotalol polytherapy) as the experimental intervention.

Comparator: The efficacy of different combinations of these drugs will be compared.

Study designs to be included: This paper will review comparative original studies (the randomized control trials, casecontrol, and cohort studies) that made a comparison between different multiple medication options as treatment of fetal supraventricular tachyarrhythmia.

Eligibility criteria: The included literatures should meet the requirements of study design, participant, intervention, comparator and outcome measure.

Information sources: PubMed, EMBASE, Cochrane Library, Web of Science, Google Scholar and ClinicalTrials.gov will be searched from inception to September 2020. A handsearching for grey literature, including unpublished conference articles will be performed.

Main outcome(s): The primary outcome will be the effect of therapy, defined as fetal rhythm conversion to sinus rhythm (SR) or rate control in those fetuses.

Quality assessment / Risk of bias analysis: The version 2 of the Cochrane risk-of bias tool for randomized trials (RoB 2) will be used to assess the risk of bias for randomized clinical trials. The NewcastleOttawa scale will be used to assess the risk of bias of case-control studies, and cohort studies.

Strategy of data synthesis: Statistical analyses will be performed using STATA software. Data that cannot be synthesized will be described qualitatively. Heterogeneity will be assessed by thex2test and $I^{2}$ statistics. Funnel plot and egger's test will be applied to detect the potential reporting biases if more than 10 studies are included.

Subgroup analysis: If appropriate data are available, subgroup analyses will be exploratory according to the research design, therapy, and different outcomes.

Sensibility analysis: Sensitivity analysis will be conducted based on the type of study, the missing data result and the methodological quality of the included studies, when studies are adequate. We will repeat the analysis after excluding trials with a high risk of bias.

Language: There are no restrictions on the language and ethnicity of the country.

Country(ies) involved: China.

Keywords: fetal tachycardia, supraventricular tachycardia, atrial flutter, treatment, systematic review, metaanalysis.

\section{Contributions of each author:}

Author 1 - Tingting Chen - The author contributed to the development of the conceptualization, the data curation, the formal analysis, the methodology, the software and the writing - original draft.

Author 2 - Yanfeng Yang - The author contributed to the development of the data curation and the formal analysis.

Author 3 - Kun Shi - The author contributed to the development of the formal analysis and the methodology. 
Author 4 - Yue Pan - The author contributed to the development of the methodology.

Author 5 - Sumei Wei - The author contributed to the development of the software.

Author 6 - Zexuan Yang - The author contributed to the development of the software.

Author 7 - Xiao Yang - The author contributed to the development of the funding acquisition, the supervision and the Writing - review \& editing. 